\title{
CorMatrix Anterior Leaflet Augmentation of the Tricuspid Valve: Midterm Results
}

\author{
Giuseppe Rescigno, MD, FETCS,${ }^{1}$ Sandeep S. Hothi, PhD, FRCP, ${ }^{2}$ Christopher Bond, MD,${ }^{1}$ \\ Mauin Uddin, MRCS Ed, ${ }^{1}$ Veena Bhatti, $\mathrm{MSc}^{3}$ John Stephen Billing, PhD, FRCS ${ }^{1}$ \\ ${ }^{1}$ Cardiothoracic Department, ${ }^{2}$ Cardiology Department, and ${ }^{3}$ Cardiac Investigation Department, Heart and Lung Centre, \\ Royal Wolverhampton NHS Trust, Wolverhampton, UK
}

\section{ABSTRACT}

Background: Tricuspid annuloplasty is the most common surgical approach to correct tricuspid regurgitation (TR). In some patients, however, anterior leaflet patch augmentation may be necessary to optimize tricuspid competence. We reviewed our center cohort over the midterm and long term.

Methods: From January 2013 to August 2018, 424 tricuspid valve procedures were performed, of which 420 were repairs and 4 were replacements. Indications were either isolated severe TR or moderate or greater TR, concomitant with other surgery. In the repair cohort, we identified those that had a patch augmentation, and the database was interrogated for preoperative characteristics. The resulting patients had outpatient assessment (clinical and echocardiography) at 6 weeks and at a later interval. Additionally, a comparison was made between those who had good and poor results (moderate or greater TR or cardiac death).

Results: In the repair cohort, 19 patients underwent complex tricuspid valve repair with CorMatrix anterior leaflet augmentation. Preoperative characteristics were as follows: age, $65.5 \pm 13.5$ years; New York Heart Association (NYHA) class, $3.5 \pm 0.5$; left ventricular ejection fraction, $48.3 \% \pm$ $5.9 \%$; tricuspid annular plane systolic excursion, $17.1 \pm 3.7$ $\mathrm{mm}$; right ventricle (good, mild, moderate, poor), 10, 5, 4, 0; annulus size, $40.9 \pm 6.9 \mathrm{~mm}$; mean tethering distance, $1.00 \pm$ $0.3 \mathrm{~cm}$; and mean tethering area, $1.53 \pm 1.16 \mathrm{~cm}^{2}$. Mean follow-up was $2.1 \pm 1.9$ years, and survival at 2 years was $73.8 \%$. There were 2 in-hospital deaths. Mean NYHA class was 1.0 \pm 0.5 ( 6 weeks) and $1.5 \pm 0.6$ (later follow-up); mean residual TR grade was $0.5 \pm 0.6$ ( 6 weeks) and $1.3 \pm 1.4$ (follow-up). Ten of 13 survivors had a good result at last follow-up (TR 0 to 1). We compared the preoperative and operative data of this group versus those with poor results (TR $>1$ or cardiac mortality). Significant univariate predictors of poor results were larger preoperative tethering area $(1.18 \pm 0.43$ versus $\left.2.4 \pm 1.5 \mathrm{~cm}^{2} ; P=.02\right)$, longer tethering distance $(0.87 \pm 0.21$

Received Fanuary 9, 2021; accepted fanuary 22, 2021.

Correspondence: Giuseppe Rescigno, MD, Consultant Cardiac Surgeon, Cardiothoracic Surgery Department, New Cross Hospital, Wolverhampton Road, Wolverhampton WV10 0QP, UK (e-mail: giuseppe.rescigno@nbs.net). versus $1.2 \pm 0.19 \mathrm{~cm} ; P=.007)$, or the presence of mild or greater TR at 6 weeks $(0.2 \pm 0.4$ vs $1.25 \pm 0.5 ; P=.03)$.

Conclusions: CorMatrix anterior leaflet augmentation produces successful, stable repair in the majority of this complex population. The presence of even mild TR at 6 weeks' follow-up predicts a poor result. When the tethering area or the tethering distance is significantly high, replacement is probably a better option.

\section{INTRODUCTION}

Tricuspid regurgitation (TR) is a relatively common finding [Yang 2019]. The majority of cases of tricuspid regurgitation are functional (Figure 1) [Besler 2018], with the underlying mechanism being a dilated annulus, tethering of the leaflets, or both. It has been demonstrated that moderate or greater TR is an independent predictor of decreased longevity [Nath 2004]. Severe tricuspid regurgitation should always be addressed when operating for concomitant valvular disease. When severe TR is isolated, surgery is indicated if right ventricular systolic function is not compromised. For less than severe regurgitation, correction is advised if the annular dimension is $>40 \mathrm{~mm}$ or $>21 \mathrm{~mm} / \mathrm{m}^{2}$ [Baumgartner 2017].

Historically, isolated annuloplasty is the predominant approach to treat tricuspid regurgitation. Some annuloplasty techniques, such as De Vega, produce adequate results in many patients. Unfortunately, the outcome can be disappointing in

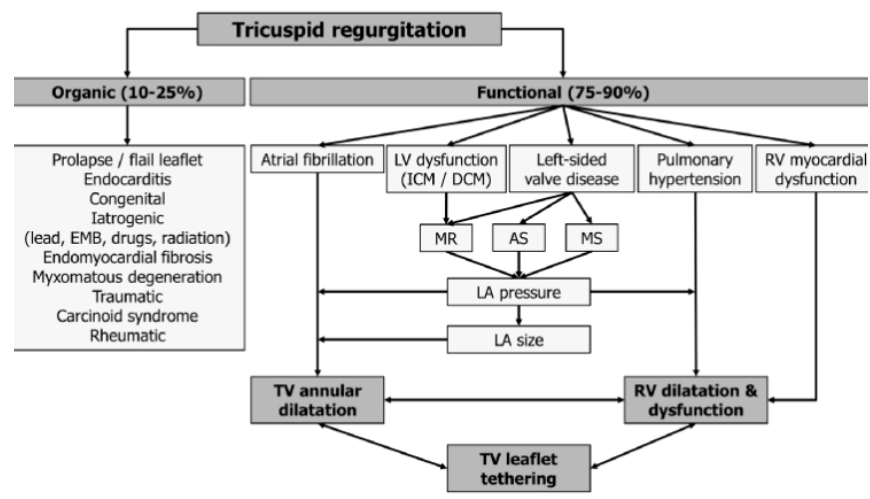

Figure 1. The possible etiologies of organic and functional tricuspid regurgitation. (Reprinted with permission from Besler [2018].) 
severe regurgitation, as the rate of long-term recurrence is high [Rivera 1985]. Over the last 3 decades, it has become clear that a prosthetic ring annuloplasty produces superior results to suture annuloplasty [McCarthy 2004].

Unfortunately, some cases of functional tricuspid regurgitation are difficult to correct with a simple annuloplasty [Roshanali 2010]. This is often due to extreme tethering of the leaflets. Replacement with a biological or mechanical prosthesis offers one remediary approach in such cases; however, it comes with attendant risks of complete heart block, valve thrombosis, and endocarditis. Although there is a paucity of strong evidence that repair is superior to replacement, consensus favors repair [Singh 2006; Wong 2020].

Anterior leaflet patch augmentation (ALA) is a relatively simple procedure that has been shown to improve the success rate of repair [Dreyfus 2008]. The ideal material to perform patch augmentation is not defined. Surgeons have several options, each with drawbacks. The most commonly used is an autologous pericardial patch [Roshanali 2010]. However, fresh autologous pericardium is prone to shrinkage; glutaraldehyde-fixed pericardium is more stable but still may degenerate over time [Kumar 1995]. Other materials have been tested and used over the years, with mixed results. One theoretically promising substitute is an extracellular matrix (ECM) derived from decellularized porcine small intestine submucosa (CorMatrix Cardiovascular, Roswell, GA). It may act as a scaffold for tissue regeneration that could potentially facilitate constructive tissue remodeling with endothelialization and connective tissue organization [Ropcke 2018; Mosala Nezhad 2019]. Unfortunately, outcomes of clinical studies to date have not always been positive [Mosala Nezhad 2016], with a relatively high incidence of failure, in particular when used to construct valved conduits [van Rijswijk 2020].

In our department, we use CorMatrix patches for rightsided defects. Our general feeling has been positive so far. The aim of the present study was therefore to review the mid- and long-term results in our cardiothoracic center of anterior leaflet augmentation of the tricuspid valve with an ECM patch.

\section{METHODS}

Our institution enrolls all patients undergoing cardiac surgery into a database (Patient Analysis and Tracking System [PATS]; Dendrite Clinical Systems, London, UK), contemporaneously recording a comprehensive set of variables. From January 2013 to August 2018, 424 tricuspid valve procedures were performed, of which 420 were repairs and 4 were replacements. In the repair cohort, we identified those with patch augmentation, and the database was interrogated for preoperative characteristics. These represented our study group.

All patients underwent preoperative, 6-week postoperative, and long-term follow-up (October 2019) transthoracic echocardiography by an accredited sonographer, using Philips Epiq and Philips IE33 systems and an S5-1 transducer (Philips UK, Guildford, UK). Additionally, an intraoperative transesophageal echocardiogram was taken in all patients to guide repair and assess competence of repair, using Philips Epiq and Philips IE33 systems and an X7-2t transducer. Echocardiographic images were analyzed on-cart or off-cart using Philips Xcelera software (Philips UK). Tricuspid regurgitation was graded as 0 , nil or trace; 1 , mild; 2 , mild to moderate; 3, moderate; or 4, severe, per international guidance for TR severity assessment [Lancellotti 2013]. All echocardiographic images were reviewed by a senior consultant cardiologist, with particular attention to tricuspid valve structure and function, including fibrosis and retraction or expansion of the patch. Clinical assessment was performed in an outpatient setting 6 weeks after surgery and at long-term follow up in October 2019.

The decision to undertake ALA was based on preoperative echocardiographic considerations and intraoperative findings. Surgery was performed on cardiopulmonary bypass using cold blood cardioplegic arrest. In all patients, a large CorMatrix patch (CorMatrix Cardiovascular) was used to augment the anterior leaflet of the tricuspid valve. The details of the tricuspid procedure have been described elsewhere [Rescigno 2019].

Continuous variables are summarized as mean \pm standard deviation (SD), and categorical variables as count and percentage. Univariate analysis was undertaken to determine predictors of poor echocardiographic results at follow-up using $t$ test or $\chi^{2}$ test as appropriate. An $\alpha$ value of 0.05 was considered significant. All analysis was performed with Prism 7 (GraphPad, San Diego, CA).

\section{RESULTS}

Nineteen patients undergoing anterior leaflet CorMatrix patch augmentation were identified from our database. Five $(26 \%)$ underwent isolated tricuspid valve surgery, and $14(74 \%)$ underwent concomitant surgery on another valve. Mean age was $65.5 \pm 13.5$ years; 17 (89\%) were female. Demographic and preoperative characteristics are summarized in Table 1 . The patients were all highly symptomatic (52.6\% were New York Heart Association [NYHA] class IV on admission). Mean Euroscore I was $6.6 \pm 2.8$. Twelve patients were in permanent atrial fibrillation (63.1\%). Intraoperative findings demonstrated that the etiology was functional in all patients. However, 6 of 19 patients $(31.6 \%)$ had left-sided lesions of rheumatic etiology; therefore, we cannot completely exclude microscopic features of this disease on the tricuspid valve.

Echocardiographic features are summarized in Table 2. We used a 4-grade system to describe the severity of valvular regurgitation. Briefly, tricuspid regurgitation was severe in 17 patients and moderate to severe in the remaining 2 patients. Right ventricle (RV) function was normal in all patients but 2 , in whom it was moderate; this was confirmed by the mean tricuspid annular plane systolic excursion $(17.2 \pm 3.7 \mathrm{~mm})$. Mean tricuspid annulus (TA) dimension was increased (40.9 $\pm 6.9 \mathrm{~mm}$ ); however, the mechanism of regurgitation was also related to $\mathrm{RV}$ remodeling and papillary muscle tethering. 
Table 1. Preoperative Characteristics*

\begin{tabular}{lc}
\hline Characteristic & Value \\
\hline Age $(y)$ & $65.5 \pm 13.5$ \\
Sex & \\
Male & 2 \\
Female & 17 \\
Euroscore I & $6.65 \pm 2.80$ \\
NYHA class & $3.53 \pm 0.51$ \\
I & 0 \\
II & 0 \\
III & $9(47)$ \\
IV & $10(53)$ \\
Rhythm & \\
Sinus rhythm & $7(37)$ \\
Atrial fibrillation & $12(63)$ \\
Redo surgery & $2(10.5)$ \\
\hline
\end{tabular}

*Data are mean $\pm S D, n$, or $n(\%)$.

Thus, the mean tethering area was $1.53 \pm 1.16 \mathrm{~cm}^{2}$, and the tethering distance was $1.00 \pm 0.30 \mathrm{~cm}$.

Two in-hospital deaths were recorded. One patient suffered iatrogenic aortic dissection, related to aortic cannulation. Aortic dissection repair was performed in addition to the planned mitral and tricuspid valve repairs. This patient died in intensive therapy unit with multiorgan failure, probably due to dissection-related hypoperfusion. The second patient developed nonconvulsive status epilepticus; no specific etiology was discovered. The remainder of patients had, in general, an uneventful postoperative course. In 1 patient, drainage of pericardial effusion was necessary.

A breakdown of the operations performed is presented in Table 3. At 6-week outpatient follow-up, clinical status was satisfactory. Mean NYHA class was reduced from baseline, at $1.06 \pm 0.57$. Residual tricuspid regurgitation was absent in 8 patients and mild in a further 8 . In the remaining patient, 6-week echocardiogram showed mild to moderate TR. Classified in accordance with the grading system outlined above, the mean TR grade was $0.56 \pm 0.64$.

The duration of late follow-up was $2.09 \pm 1.7$ years. Three deaths were recorded. One patient died from complications of Guillain-Barré syndrome; in the other 2 patients, the recorded cause of death was cardiovascular. Survival at 2 years was therefore $73.8 \%$. Figure 2 shows the observed actuarial survival curve. The mean clinical status of patients was significantly reduced (mean NYHA $1.53 \pm 0.65$ ) compared with the 6-week review $(P=.016)$ (Figure 3). From an echocardiographic perspective, the mean residual regurgitation grade also increased from the short-term assessment $(1.29 \pm 1.36 ; P=.03)$ (Figure 4). No significant abnormalities of the patch in terms of fibrosis, retraction, expansion, or detachment were detected.
Table 2. Preoperative Echocardiographic Findings*

\begin{tabular}{lc}
\hline Characteristic & Value \\
\hline Left ventricular ejection fraction (\%) & $48.5 \pm 6.45$ \\
Right ventricular ejection fraction & \\
$\quad$ Normal & $9(47.3)$ \\
Mild & $6(31.6)$ \\
Moderate & $4(21.1)$ \\
Severe & 0 \\
Pulmonary artery systolic pressure & $44.6 \pm 11.6$ \\
(mmHg) & $17.2 \pm 3.74$ \\
Tricuspid annular plane excursion & \\
(mm) & \\
Tricuspid regurgitation severity & $17(89)$ \\
4 & $2(11)$ \\
3 & 0 \\
2 & 0 \\
1 & 0 \\
0 & $1.53 \pm 1.16$ \\
Tricuspid annulus diameter (mm) & $1.00 \pm 0.30$ \\
Tethering area (cm2) & \\
Tethering distance (mm) & \\
\hline
\end{tabular}

*Data are mean \pm SD or $\mathrm{n}(\%)$.

Table 3. Operations Performed

\begin{tabular}{lc}
\hline Valves intervened & $\mathrm{n}(\%)$ \\
Isolated tricuspid & $6(31.6)$ \\
Tricuspid and mitral & $8(42.1)$ \\
Tricuspid, aortic, and mitral & $5(22.2)$
\end{tabular}

Subsequently, we divided the population into 2 groups according to the repair result at long-term follow-up. Group A $(n=10)$ included patients with a good echocardiography result (residual regurgitation nil or mild), and group $\mathrm{B}$ ( $\mathrm{n}=$ 6) included subjects with a poor result (residual regurgitation above mild or cardiovascular death). The univariate analysis showed that factors affecting the long-term echocardiography result were preoperative tethering area $(1.18 \pm 0.43$ versus $\left.2.40 \pm 1.57 \mathrm{~cm}^{2} ; P=.02\right)$, tethering distance $(0.87 \pm$ 0.21 versus $1.2 \pm 0.19 \mathrm{~cm} ; P=.007)$, and 6 -week residual TR grade mild or above $(0.2 \pm 0.4$ versus $1.25 \pm 0.5 ; P=.03)$.

\section{DISCUSSION}

The results of our relatively small series of patients show that CorMatrix patch augmentation could be a viable solution 


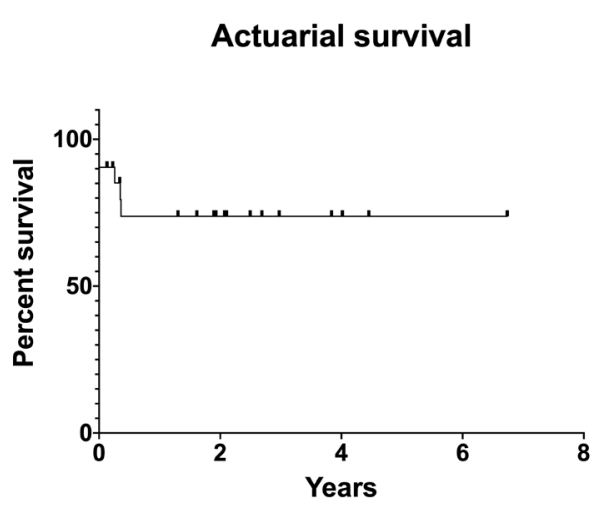

Figure 2. Survival curve of patients with anterior leaflet augmentation. Survival at 2 years was $73.8 \%$.

to improve functional tricuspid repair outcome in selected patients. Survival at 2 years was good, considering the highrisk profile of many patients. The majority of patients had a good echocardiographic result at follow-up. This partly contradicts other recent reports about the use of CorMatrix as an augmentation patch in the tricuspid position. Indeed, we had no clear failures related to the patch itself. We observed that patients who showed a poor echocardiographic outcome at longer-term follow-up were those with significant tethering of the leaflets at baseline or a suboptimal TR grade 6 weeks after surgery. We speculate that this might indicate a technical problem or poor candidate selection.

\section{Clinical Outcomes}

Because of the limited number of patients, it is difficult to draw definite conclusions. Hospital mortality was high but related to complications that were not directly linked to the tricuspid valve procedure itself. One patient died from iatrogenic, intraoperative aortic dissection, and another from a neurological complication. The 2-year survival was good, if we consider that many patients were extremely sick, having a high NYHA class preoperatively. Before surgery, they frequently had dyspnea at rest and were on loop diuretics. Pulmonary hypertension was also a common finding. In assessing our results, it should be noted that patients with severe tricuspid regurgitation have been shown to have a 1-year survival rate of $63 \%$ regardless of left ventricular ejection fraction or pulmonary artery pressure [Nath 2004]. At late follow-up, most of our patients had no or mild symptoms, regardless of echocardiographic outcome. In many patients, this was of course due to the simultaneous correction of other valvular disease; therefore, the clinical benefits are not fully attributable to the tricuspid repair. Only 1 patient complained of shortness of breath during everyday activities.

\section{Echocardiographic Outcomes}

These tricuspid valves were highly dysfunctional and presented significant challenges to conventional repair. Almost all were severely regurgitant; the annulus was frequently severely dilated, and there was also a significant depth and area of tethering. Therefore, it is considered unlikely that a

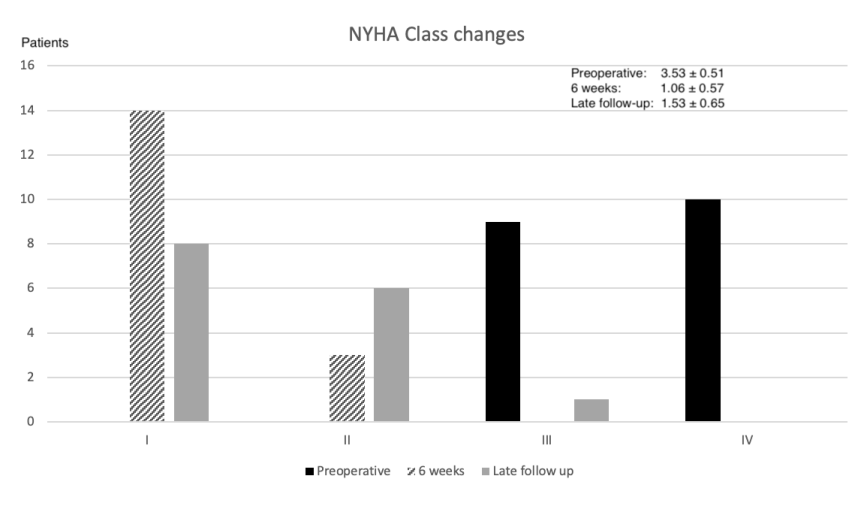

Figure 3. NYHA class severity over time.

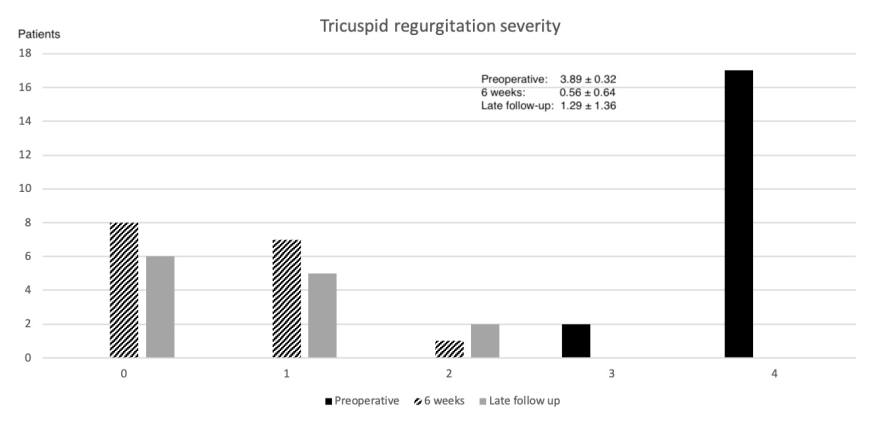

Figure 4. Tricuspid regurgitation over time.

simple annuloplasty would have produced adequate valvular coaptation and therefore durable competence. The ALA was a viable solution for these patients. Early echocardiographic results were good. The 6-week follow-up assessment demonstrated no or mild regurgitation in almost every patient. At late follow-up, we observed mixed results. The majority of patients showed a good outcome. There were 10 patients with no or mild tricuspid regurgitation. In 4 patients, however, there was significant recurrence of valvular regurgitation. The last echocardiograms of the 2 deceased patients with a cardiovascular cause were also suboptimal. Therefore, 6 patients had a poor outcome, whether echocardiographically, clinically, or both.

Dreyfus et al [2008] were the first to publicize ALA to treat functional tricuspid regurgitation. In a series similar to ours, comprising 15 patients, they had an excellent intraoperative result, with no residual regurgitation and good coaptation length. However, unlike the present report, they had no formal follow-up. It can be speculated that some of their patients could have experienced some form of recurrence later on. In any case, we speculate that outcomes are heavily dependent on candidate selection. Our findings prompt a careful assessment of preoperative echocardiography before planning an ALA plus undersized annuloplasty ring. Roshanali et al [2010] proposed an interesting decision-making protocol to plan additional ALA to ring annuloplasty. In their series, patients randomly assigned to ALA when the tethering distance was $>8 \mathrm{~mm}$ or the tethering area was $>16 \mathrm{~mm}^{2}$ had a 
better 1-year result in terms of recurrent tricuspid regurgitation. Our patients had values that met such indications for ALA. However, our case series is likely to have included patients who met these criteria for ALA but whose disease may have been too advanced, such that even this additional procedure was not able to achieve freedom from recurrent regurgitation. Accordingly, those patients of ours with poor follow-up results had an extreme tethering area, well above the threshold suggested by Roshanali et al [2010].

These considerations highlight a question for future studies. It will be important to find the threshold above which ALA repair is formally contraindicated (and a replacement should be performed instead). The 6-week postoperative echo was already able to predict a poor late result. Patients with even mild recurrent regurgitation at this stage had a significant leak at follow-up or died from cardiovascular causes. Studies about functional mitral regurgitation have clearly demonstrated this phenomenon [Calafiore 2011]. Consequently, the aim of a correction repair of the mitral valve is to achieve zero regurgitation with a good coaptation area, to avoid any disappointment at follow-up. We believe that this lesson applies to functional tricuspid regurgitation as well. The lack of a proper and wide coaptation could be a factor influencing long-term poor results.

\section{CorMatrix Patch}

The majority of published articles about the use of patches for atrioventricular valves in adult patients refer to mitral valve procedures. The use of patches for tricuspid valve repair is less frequent [Wallen 2014]. The ideal material to patch valves is not established. In our series, we used CorMatrix, as this potentially has several advantages over routine pericardial patches. Theoretically, it would act as a scaffold on which autologous tissue may grow. However, there are reports of failure and concerns about the CorMatrix patch. Tomdio et al [2018] even reported a case of dehiscence and embolization of a CorMatrixderived tricuspid valve. On the left side of the heart, there have also been disappointing results, with early and late failures of mitral patches [Kelley 2017]. This scaffold relies on inflammation to trigger regenerative mechanisms. However, the timing of this process might not be ideal, and inflammatory events could potentially disrupt remodeling [Mosala Nezhad 2016].

In our series, the observed failures were probably unrelated to the material used. In fact, from the above considerations, the initial tricuspid recurrence was already present in the early postsurgical follow-up period. Our follow-up echocardiography assessment was particularly focused on finding any possible retraction or billowing of the patch, as these are the 2 common features of failure. We can affirm that none of the patients showed these signs. To summarize, we believe that the CorMatrix patch used for our ALA procedures did not show significant degeneration during follow-up. Obviously, a longer observation period is mandatory before reaching a solid conclusion.

\section{Limitations of the Study}

This is a small series with all related constraints. The statistical inference is therefore limited, and we could not perform a multivariate analysis. Many patients had combined surgical procedures. The clinical effect of the correction of multiple valve diseases is confounding.

\section{CONCLUSIONS}

Anterior leaflet augmentation of the tricuspid valve is a relatively simple procedure that increases the chance of a successful and enduring repair. The CorMatrix patch has shown good midterm results. Careful preoperative echo assessment is necessary to plan the correct operation. An early residual leak is a predictor of poor midterm result. Further research is required to confirm these findings in a wider population and to refine patient selection to identify those most likely to benefit and those with advanced disease who are unlikely to benefit.

\section{REFERENCES}

Baumgartner H, Falk V, Bax JJ, et al 2017 ESC/EACTS Guidelines for the management of valvular heart disease. Eur Heart J 2017;38:2739-2791.

Besler C, Seeburger J, Thiele H, Lurz P. Treatment options for severe functional tricuspid regurgitation: Indications, techniques, and current challenges. e-J Cardiol Pract 2018;16. Available at: https://www.escardio. org/Journals/E-Journal-of-Cardiology-Practice/Volume-16/Treatmentoptions-for-severe-functional-tricuspid-regurgitation-indications-techniques-and-current-challenges.

Calafiore AM, Iacò AL, Bivona A, et al. Echocardiographically based treatment of chronic ischemic mitral regurgitation. J Thorac Cardiovasc Surg 2011;141:1150 1156.

Dreyfus GD, Raja SG, John Chan KM. Tricuspid leaflet augmentation to address severe tethering in functional tricuspid regurgitation. Eur J Cardiothorac Surg 2008;34:908-910.

Kelley TM, Kashem M, Wang H, et al. Anterior leaflet augmentation with CorMatrix porcine extracellular matrix in twenty-five patients: Unexpected patch failures and histologic analysis. Ann Thorac Surg 2017;103:114-120.

Kumar SP, Prabhakar G, Kumar M, Kumar N, Shahid M, Ali ML. Comparison of fresh and glutaraldehyde-treated autologous stented pericardium as pulmonary valve replacement. J Card Surg 1995;10:545-551.

Lancellotti P, Tribouilloy C, Hagendorff A, et al. Recommendations for the echocardiographic assessment of native valvular regurgitation: An executive summary from the European Association of Cardiovascular Imaging. Eur Heart J Cardiovasc Imaging 2013;14:611-644.

McCarthy PM, Bhudia SK, Rajeswaran J, et al. Tricuspid valve repair: Durability and risk factors for failure. J Thorac Cardiovasc Surg 2004;127:674-685.

Mosala Nezhad Z, Poncelet A, de Kerchove L, Gianello P, Fervaille C, El Khoury G. Small intestinal submucosa extracellular matrix (CorMatrix $\left.{ }^{\circledR}\right)$ in cardiovascular surgery: A systematic review. Interact Cardiovasc Thorac Surg 2016;22:839-850.

Mosala Nezhad Z, Poncelet A, Fervaille C, Gianello P. Comparing the host reaction to CorMatrix and different cardiac patch materials implanted subcutaneously in growing pigs. Thorac Cardiovasc Surg 2019;67:44-49. 
Nath J, Foster E, Heidenreich PA. Impact of tricuspid regurgitation on long-term survival. J Am Coll Cardiol 2004;43:405-409.

Rescigno G, Meraglia A, Uddin M, Billing JS. CorMatrix patch augmentation of the tricuspid valve anterior leaflet. October 2019. Available at: https://ctsnet.figshare.com/articles/media/Cormatrix_Patch_Augmentation_of_the_Tricuspid_Valve_Anterior_Leaflet/9983360.

Rivera R, Duran E, Ajuria M. Carpentier's flexible ring versus De Vega's annuloplasty. A prospective randomized study. J Thorac Cardiovasc Surg 1985;89:196-203.

Ropcke DM, Rasmussen J, Ilkjær C, Skov SN, Tjørnild MJ, Baandrup UT, et al. Mid-term function and remodeling potential of tissue engineered tricuspid valve: Histology and biomechanics. J Biomech 2018;71:52-58.

Roshanali F, Saidi B, Mandegar MH, Yousefnia MA, Alaeddini F. Echocardiographic approach to the decision-making process for tricuspid valve repair. J Thorac Cardiovasc Surg 2010;139:1483-1487.

Singh SK, Tang GH, Maganti MD, Armstrong S, Williams WG,
David TE, Borger MA. Midterm outcomes of tricuspid valve repair versus replacement for organic tricuspid disease. Ann Thorac Surg 2006;82:1735-1741.

Tomdio AN, Moey MYY, Siddiqui I, Movahed A. Dehiscence and embolization of CorMatrix tricuspid valve replacement in the setting of infective endocarditis: a case report. Eur Heart J Case Rep 2018;2:yty086.

van Rijswijk JW, Talacua H, Mulder K, et al. Failure of decellularized porcine small intestinal submucosa as a heart valved conduit. J Thorac Cardiovasc Surg 2020;160:e201-e205.

Wallen J, Rao V. Extensive tricuspid valve repair after endocarditis using CorMatrix extracellular matrix. Ann Thorac Surg 2014;97:1048-1050.

Wong WK, Chen SW, Chou AH, et al. Late outcomes of valve repair versus replacement in isolated and concomitant tricuspid valve surgery: A nationwide cohort study. J Am Heart Assoc 2020;9:e015637.

Yang L, Chen H, Pan W, et al. Analyses for prevalence and outcome of tricuspid regurgitation in China: An echocardiography study of 134,874 patients. Cardiology 2019;142:40-46. 